PROCEEDINGS OF THE

AMERICAN MATHEMATICAL SOCIETY

Volume 138, Number 3, March 2010, Pages 799-808

S 0002-9939(09)10084-9

Article electronically published on October 20, 2009

\title{
IRRATIONALITY EXPONENT AND RATIONAL APPROXIMATIONS WITH PRESCRIBED GROWTH
}

\author{
STÉPHANE FISCHLER AND TANGUY RIVOAL
}

(Communicated by Ken Ono)

\begin{abstract}
Let $\xi$ be a real irrational number. We are interested in sequences of linear forms in 1 and $\xi$, with integer coefficients, which tend to 0 . Does such a sequence exist such that the linear forms are small (with given rate of decrease) and the coefficients have some given rate of growth? If these rates are essentially geometric, a necessary condition for such a sequence to exist is that the linear forms are not too small, a condition which can be expressed precisely using the irrationality exponent of $\xi$. We prove that this condition is actually sufficient, even for arbitrary rates of growth and decrease. We also make some remarks and ask some questions about multivariate generalizations connected to Fischler-Zudilin's new proof of Nesterenko's linear independence criterion.
\end{abstract}

\section{INTRODUCTION}

In 1978, Apéry [2] proved the irrationality of $\zeta(3)$ by constructing two explicit sequences of integers $\left(u_{n}\right)_{n}$ and $\left(v_{n}\right)_{n}$ such that $0 \neq u_{n} \zeta(3)-v_{n} \rightarrow 0$ and $u_{n} \rightarrow$ $+\infty$, both at geometric rates. He also deduced from this an upper bound for the irrationality exponent $\mu(\zeta(3))$ of $\zeta(3)$. In general, the irrationality exponent $\mu(\xi)$ of an irrational number $\xi$ is defined as the infimum of all real numbers $\mu$ such that the inequality

$$
\left|\xi-\frac{p}{q}\right|>\frac{1}{q^{\mu}}
$$

holds for all integers $p, q$, with $q$ sufficiently large. It is well-known that $\mu(\xi) \geq 2$ for any irrational number $\xi$ and that it equals 2 for almost all irrational numbers. The following lemma is often used (as in Apéry's proof) to bound $\mu(\xi)$ from above, for example for the numbers $\log (2)$ and $\zeta(2)$. (Other lemmas can be used to bound the irrationality exponent of numbers of a different nature, such as $\exp (1)$.)

Lemma 1. Let $\xi \in \mathbb{R} \backslash \mathbb{Q}$, and let $\alpha, \beta$ be real numbers such that $0<\alpha<1$ and $\beta>1$. Assume there exist integer sequences $\left(u_{n}\right)_{n \geq 1}$ and $\left(v_{n}\right)_{n \geq 1}$ such that

$$
\lim _{n \rightarrow+\infty}\left|u_{n} \xi-v_{n}\right|^{1 / n}=\alpha \text { and } \limsup _{n \rightarrow+\infty}\left|u_{n}\right|^{1 / n} \leq \beta .
$$

Then we have $\mu(\xi) \leq 1-\frac{\log \beta}{\log \alpha}$.

Received by the editors June 9, 2009

2000 Mathematics Subject Classification. Primary 11J82; Secondary 11J04, 11J13, 11J72.

(C)2009 American Mathematical Society 
The proof of Lemma 1 is not difficult. Many variants of this result exist; a slightly more general version of Lemma 1 will be proved in \$4.1. Another variant, proved in [6] (Proposition 3.1), asserts that Lemma 1] holds when (1.1) is replaced with

$$
\limsup _{n \rightarrow+\infty} \frac{\left|u_{n+1} \xi-v_{n+1}\right|}{\left|u_{n} \xi-v_{n}\right|} \leq \alpha \text { and } \limsup _{n \rightarrow+\infty} \frac{u_{n+1}}{u_{n}} \leq \beta
$$

In this text, we prove that Lemma 1 and these variants are best possible by obtaining a very precise converse result:

Theorem 1. Let $\xi \in \mathbb{R} \backslash \mathbb{Q}$, and let $\alpha, \beta$ be real numbers such that $0<\alpha<1$, $\beta>1$ and $\mu(\xi)<1-\frac{\log \beta}{\log \alpha}$. Then there exist integer sequences $\left(u_{n}\right)_{n \geq 1}$ and $\left(v_{n}\right)_{n \geq 1}$ such that

$$
\lim _{n \rightarrow+\infty} \frac{u_{n} \xi-v_{n}}{\alpha^{n}}=\lim _{n \rightarrow+\infty} \frac{u_{n}}{\beta^{n}}=1
$$

and, consequently,

$$
\lim _{n \rightarrow+\infty} \frac{\left|u_{n+1} \xi-v_{n+1}\right|}{\left|u_{n} \xi-v_{n}\right|}=\lim _{n \rightarrow+\infty}\left|u_{n} \xi-v_{n}\right|^{1 / n}=\alpha
$$

and

$$
\lim _{n \rightarrow+\infty} \frac{u_{n+1}}{u_{n}}=\lim _{n \rightarrow+\infty}\left|u_{n}\right|^{1 / n}=\beta .
$$

Theorem 1 answers completely all questions asked in [6], where the density exponent is defined (see $\$ 3$ below).

An essential feature of Lemma 1 and all its variants is that the sequences $\left(u_{n}\right)$ and $\left(u_{n} \xi-v_{n}\right)$ are assumed to have essentially geometrical behaviour. An assumption of this kind is necessary, since the convergents of the continued fraction expansion of $\xi$ (for instance) always make up a sequence of approximants to $\xi$ that are far more precise, but if $\mu(\xi)>2$, they don't have geometrical behaviour.

However, a geometrical behaviour is not necessary in Theorem 1, as the following generalization shows. In this statement and throughout the paper, we denote by $o(1)$ any sequence that tends to 0 as $n$ tends to infinity.

Theorem 2. Let $\xi \in \mathbb{R} \backslash \mathbb{Q}$, and let $\left(Q_{n}\right)$ and $\left(\varepsilon_{n}\right)$ be sequences of positive real numbers with

$$
\lim _{n \rightarrow+\infty} Q_{n}=+\infty, \quad \lim _{n \rightarrow+\infty} \varepsilon_{n}=0 \quad \text { and } \quad \varepsilon_{n} \geq Q_{n}^{-\frac{1}{\mu-1}+o(1)},
$$

where $\mu$ is a real number such that $\mu>\mu(\xi)$.

Then there exist integer sequences $\left(u_{n}\right)$ and $\left(v_{n}\right)$ such that

$$
\lim _{n \rightarrow+\infty} \frac{u_{n}}{Q_{n}}=\lim _{n \rightarrow+\infty} \frac{u_{n} \xi-v_{n}}{\varepsilon_{n}}=1 .
$$

The important point in this theorem is that our only assumption is that $\varepsilon_{n}$ is not too small, namely

$$
\limsup _{n \rightarrow+\infty} \frac{-\log \varepsilon_{n}}{\log Q_{n}}<\frac{1}{\mu(\xi)-1} .
$$

Theorem [2] answers the questions asked in $\S 8$ of [6].

The structure of this text is as follows. In Section 2 we prove Theorem 2 (and therefore, as a special case, Theorem 1). Then we recall (in 93) the definition 6] of the density exponent and deduce from Theorem 1 that it is always 0 or $\infty$. In 44 we prove a slight generalization of Lemma 1 which enables us to obtain a 
general statement (containing Lemma 11 Theorem 1 and Theorem 2) consisting of the equality of several exponents of Diophantine approximation.

Finally we partially generalize (in $\$ 4.3$ ) this statement to the multivariate setting, where we consider simultaneously several real numbers $\xi_{0}, \ldots, \xi_{r}$ instead of just one $\xi$. We then explain the connection between Lemma 1 and Nesterenko's linear independence criterion [10, used in particular in the proof $([3],[1])$ that $\zeta(s)$ is irrational for infinitely many odd integers $s \geq 3$. This criterion has been recently generalized in [7] to take into account common divisors to the coefficients of the linear forms; the proof involves Minkowski's convex body theorem. In 4.3 , we write down this new proof in the case of Nesterenko's criterion only (where Minkowski's convex body theorem is simply replaced with Dirichlet's pigeonhole principle) in terms of exponents of Diophantine approximation. This enables us to make a connection with the other results of the present paper and to ask several questions in the multivariate setting.

\section{Proof of Theorem 2}

The proof of Theorem 2 is based on the following lemma, which is proved inside the proof of Lemma 7.3 of [6] (p. 39) and is the main step in the proof [6] that almost all $\xi$ (with respect to Lebesgue measure) have density exponent zero.

Lemma 2. Let $c, c^{\prime}, \varepsilon, Q$ be real numbers such that $1<c<c^{\prime}<2,0<\varepsilon<1$, and $Q>1$.

Let $\xi$ be an irrational number with $0<\xi<1$. Then (at least) one of the following assertions holds:

(i) There exist coprime integers $u \geq 1$ and $v \in\{0, \ldots, u\}$ such that

$$
u<\frac{2 c^{2}}{(c-1)\left(c^{\prime}-c\right)} \frac{1}{\varepsilon}
$$

and

$$
\left|\xi-\frac{v}{u}\right| \leq \frac{2}{c-1}\left(1+\frac{c^{2}}{c^{\prime}-c}\right) \frac{1}{u Q} .
$$

(ii) There exist integers $p$ and $q$ such that

$$
Q \leq q \leq c Q \text { and } \frac{\varepsilon}{q} \leq \xi-\frac{p}{q} \leq \frac{c^{\prime} \varepsilon}{q} .
$$

This lemma is interesting when $\varepsilon$ is much larger than $1 / Q$. It means that unless $\xi$ is very close to a rational number with denominator essentially bounded by $1 / \varepsilon$, it is possible to find a fraction $p / q$ (which may not be in its lowest terms) such that $q$ has essentially the size of $Q$, and $q \xi-p$ that of $\varepsilon$. The interesting part in proving Theorem 2 is that we obtain $Q \leq q \leq c Q$ and $\varepsilon \leq q \xi-p \leq c^{\prime} \varepsilon$, where $c$ and $c^{\prime}$ are constants that can be chosen arbitrarily close to 1 . A variant of this lemma, in which one obtains only $Q \leq q \leq 2 Q$ and $\varepsilon \leq q \xi-p \leq 3 \varepsilon$, is proved in [5] (Lemma 5). The proof uses the same ideas as those of Lemma 2 , but is fairly less complicated.

The proof [6] of Lemma 2 makes use of Farey fractions. It might be possible to prove this lemma using continued fractions.

Let us now deduce Theorem 2 . 
Proof. We may assume $0<\xi<1$. Let $\left(\eta_{n}\right)$ be a sequence of positive real numbers such that $\lim _{n \rightarrow+\infty} \eta_{n}=0$ and $\eta_{n}=\varepsilon_{n}^{o(1)}$. We let $\lambda_{n}=1+\eta_{n}, \mu_{n}=1+2 \eta_{n}$, $Q_{n}^{\prime}=\frac{Q_{n}}{\sqrt{\lambda_{n}}}$ and $\varepsilon_{n}^{\prime}=\frac{\varepsilon_{n}}{\sqrt{\mu_{n}}}$. For $n$ sufficiently large, Lemma 2 applies with $c=\lambda_{n}$, $c^{\prime}=\mu_{n}, \varepsilon=\varepsilon_{n}^{\prime}$, and $Q=Q_{n}^{\prime}$. If (i) holds in this lemma and $n$ is sufficiently large, then we obtain integers $u_{n}$ and $v_{n}$ such that

$$
\left|\xi-\frac{v_{n}}{u_{n}}\right| \leq \frac{2}{\eta_{n}}\left(1+\frac{\lambda_{n}^{2}}{\eta_{n}}\right) \frac{1}{u_{n} Q_{n}^{\prime}} \leq \frac{20}{\eta_{n}^{2} u_{n} Q_{n}}
$$

and

$$
u_{n}<\frac{2 \lambda_{n}^{2}}{\eta_{n}^{2}} \frac{1}{\varepsilon_{n}^{\prime}} \leq \frac{16}{\eta_{n}^{2} \varepsilon_{n}} .
$$

Since we have $\eta_{n}=\varepsilon_{n}^{o(1)}$ and $\varepsilon_{n} \geq Q_{n}^{-\frac{1}{\mu-1}+o(1)}$, these inequalities yield

$$
\left|\xi-\frac{v_{n}}{u_{n}}\right| \leq \frac{20}{\eta_{n}^{2} u_{n} Q_{n}} \leq \frac{1}{u_{n}}\left(\frac{\varepsilon_{n} \eta_{n}^{2}}{16}\right)^{\mu-1+o(1)} \leq \frac{1}{u_{n}^{\mu+o(1)}},
$$

which is possible only for finitely many values of $n$ since $\mu>\mu(\xi)$. Therefore, as soon as $n$ is sufficiently large, Assertion (ii) of Lemma2 2 holds and provides integers $p_{n}$ and $q_{n}$ such that

$$
\frac{Q_{n}}{\sqrt{\lambda_{n}}} \leq q_{n} \leq Q_{n} \sqrt{\lambda_{n}} \text { and } \frac{\varepsilon_{n}}{\sqrt{\mu_{n}}} \leq q_{n} \xi-p_{n} \leq \varepsilon_{n} \sqrt{\mu_{n}} .
$$

This concludes the proof of Theorem 2 ,

\section{Consequences for the Density exponent}

Let $\xi \in \mathbb{R} \backslash \mathbb{Q}$. For any non-decreasing sequence $\mathbf{u}=\left(u_{n}\right)_{n}$ of positive integers, let us define

$$
\alpha_{\xi}(\mathbf{u}):=\limsup _{n} \frac{\left|u_{n+1} \xi-v_{n+1}\right|}{\left|u_{n} \xi-v_{n}\right|}, \quad \beta(\mathbf{u}):=\limsup _{n} \frac{u_{n+1}}{u_{n}},
$$

where $v_{n}$ is the nearest integer to $u_{n} \xi$. We defined in [6] the density exponent $\nu(\xi)$ of $\xi$ as the infimum of the quantity $\log \sqrt{\alpha_{\xi}(\mathbf{u}) \beta(\mathbf{u})}$ when $\mathbf{u}$ ranges through the nondecreasing sequences such that $\alpha_{\xi}(\mathbf{u})<1$ and $\beta(\mathbf{u})<+\infty$ (with the convention $\nu(\xi)=+\infty$ if there is no such $\mathbf{u})$.

We proved in [6] that $\nu(\xi)=+\infty$ when $\xi$ is a Liouville number, i.e., when $\mu(\xi)=+\infty$ (that is, when for any $\mu>0$, there exists a rational number $p / q$ such that $\left.|\xi-p / q|<1 / q^{\mu}\right)$. Theorem 2 implies the converse statement in a more precise form:

Theorem 3. If $\xi \in \mathbb{R} \backslash \mathbb{Q}$ is not a Liouville number, then $\nu(\xi)=0$.

Indeed, we may choose in Theorem 1 values of $\alpha$ and $\beta$ arbitrarily close to 1 so that the product $\alpha \beta$ is also arbitrarily close to 1 . In a sense, this annihilates the interest of $\nu(\xi)$, since it takes only two values $(0$ and $+\infty)$ and distinguishes only Liouville numbers from the other irrational numbers. However, the ideas of $[6]$ are at the base of the results presented in the present paper.

Let us make precise here what we expected in [6]. We hoped to define a quantity that would enable us to distinguish between periods (in the sense of [8]) and other numbers. In particular, we computed upper bounds for $\nu(\xi)$, for many examples of $\xi$ which are periods (see also [1]). But we did not really take into account another property of the approximations used for this: they all satisfy a linear recursion of 
finite order with polynomial coefficients of a special kind. Indeed, in all the examples of [6], the sequences $\left(u_{n}\right)_{n}$ as well as $\left(v_{n}\right)_{n}$ are such that the power series $\sum_{n \geq 0} u_{n} z^{n}$ and $\sum_{n \geq 0} v_{n} z^{n}$ are $G$-function 11 satisfying the same minimal differential equation. This is a very strong property that is not satisfied (in general) by the sequences $\left(u_{n}\right)_{n}$ and $\left(v_{n}\right)_{n}$ constructed by means of Lemma 2 to prove Theorem 1 .

\section{EXPONENTS OF DIOPHANTINE APPROXIMATION}

In this section, we state the results of this paper in terms of exponents of Diophantine approximation. This enables us to explain the connection with Nesterenko's linear independence criterion [10] and to ask some questions about multivariate generalizations of our results.

4.1. A generalization of Lemma 1, We start with a generalization of the usual Lemma 1. We do not write down the proof of this proposition because it is a special case of the upper bound $\tau_{r}(\underline{\xi}) \leq \frac{1}{\omega_{0}(\underline{\xi})}$ proved in Theorem 5 (see $₫ \underline{4.3}$ ). To deduce Lemma 1 from this proposition, one takes $\tau=-\frac{\log \alpha}{\log \beta}$ and uses the fact that $\lim _{n \rightarrow+\infty} \frac{\log \left|u_{n} \xi-v_{n}\right|}{n}=\log \alpha$ implies $\lim _{n \rightarrow+\infty} \frac{\log \left|u_{n+1} \xi-v_{n+1}\right|}{\log \left|u_{n} \xi-v_{n}\right|}=1$.

Proposition 1. Let $\xi \in \mathbb{R} \backslash \mathbb{Q}$ and $\tau>0$. Assume there exist integer sequences $\left(u_{n}\right)$ and $\left(v_{n}\right)$ with $u_{n} \neq 0$ for any $n$ and such that

$$
u_{n} \xi-v_{n} \rightarrow 0,\left|u_{n+1} \xi-v_{n+1}\right|=\left|u_{n} \xi-v_{n}\right|^{1+o(1)} \text {, and }\left|u_{n} \xi-v_{n}\right| \leq\left|u_{n}\right|^{-\tau+o(1)} \text {. }
$$

Then we have $\mu(\xi) \leq 1+\frac{1}{\tau}$.

4.2. The univariate case. Let $\xi$ be an irrational real number. Let us consider the following sets:

- $\mathcal{T}(\xi)$ is the set of all $\tau>0$ for which there exist integer sequences $\left(u_{n}\right)$ and $\left(v_{n}\right)$ with $u_{n} \neq 0$ for any $n$, and

$$
u_{n} \xi-v_{n} \rightarrow 0,\left|u_{n+1} \xi-v_{n+1}\right|=\left|u_{n} \xi-v_{n}\right|^{1+o(1)} \text {, and }\left|u_{n} \xi-v_{n}\right| \leq\left|u_{n}\right|^{-\tau+o(1)} \text {. }
$$

- $\mathcal{T}^{\prime}(\xi)$ is the set of all $\tau>0$ for which there exist integer sequences $\left(u_{n}\right)$ and $\left(v_{n}\right)$, and $0<\alpha<1<\beta$, with

$$
\left|u_{n} \xi-v_{n}\right|^{1 / n} \rightarrow \alpha, \quad \limsup _{n \rightarrow+\infty}\left|u_{n}\right|^{1 / n} \leq \beta, \quad \text { and } \quad \tau=\frac{-\log \alpha}{\log \beta}
$$

- $\mathcal{T}^{\prime \prime}(\xi)$ is the set of all $\tau>0$ such that, for any increasing sequence $\left(Q_{n}\right)$ of positive integers there exist integer sequences $\left(u_{n}\right)$ and $\left(v_{n}\right)$ with

$$
\left|u_{n}\right| \leq Q_{n}^{1+o(1)} \quad \text { and } \quad\left|u_{n} \xi-v_{n}\right|=Q_{n}^{-\tau+o(1)} .
$$

- $\mathcal{T}^{\prime \prime \prime}(\xi)$ is the set of all $\tau>0$ such that, for any sequences $\left(Q_{n}\right)$ and $\left(\varepsilon_{n}\right)$ of positive real numbers with

$$
\lim _{n \rightarrow+\infty} Q_{n}=+\infty, \quad \lim _{n \rightarrow+\infty} \varepsilon_{n}=0 \quad \text { and } \quad \varepsilon_{n} \geq Q_{n}^{-\tau+o(1)},
$$

there exist integer sequences $\left(u_{n}\right)$ and $\left(v_{n}\right)$ with

$$
\lim _{n \rightarrow+\infty} \frac{u_{n}}{Q_{n}}=\lim _{n \rightarrow+\infty} \frac{u_{n} \xi-v_{n}}{\varepsilon_{n}}=1 .
$$

\footnotetext{
${ }^{1}$ A power series $\sum_{n>0} a_{n} z^{n} \in \mathbb{Q}[[z]]$ is a $G$-function when: 1) it satisfies a linear differential equation, 2) it has a finite positive radius of convergence, 3 ) the least common multiple of the denominators of $a_{0}, a_{1}, \ldots, a_{n}$ is bounded by $C^{n}$ for some $C>0$.
} 
Theorem 4 below shows that $\tau \leq 1$ for any $\tau$ in $\mathcal{T}(\xi)$ (resp. $\left.\mathcal{T}^{\prime}(\xi), \mathcal{T}^{\prime \prime}(\xi), \mathcal{T}^{\prime \prime \prime}(\xi)\right)$.

We let

$$
\tau(\xi)=\sup \mathcal{T}(\xi)
$$

and in the same way $\tau^{\prime}(\xi)=\sup \mathcal{T}^{\prime}(\xi), \tau^{\prime \prime}(\xi)=\sup \mathcal{T}^{\prime \prime}(\xi), \tau^{\prime \prime \prime}(\xi)=\sup \mathcal{T}^{\prime \prime \prime}(\xi)$, with the convention $\sup \emptyset=0$, so that each of $\tau(\xi), \tau^{\prime}(\xi), \tau^{\prime \prime}(\xi), \tau^{\prime \prime \prime}(\xi)$ belongs to $[0,1]$.

If we have $0<\tau<\tau^{\prime}$ and $\tau^{\prime} \in \mathcal{T}(\xi)$, then $\tau \in \mathcal{T}(\xi)$ so that $\mathcal{T}(\xi)$ is $\emptyset$, $(0, \tau(\xi)$ ] or $(0, \tau(\xi))$. The same holds for $\mathcal{T}^{\prime}(\xi), \mathcal{T}^{\prime \prime}(\xi), \mathcal{T}^{\prime \prime \prime}(\xi)$.

Moreover the inclusions $\mathcal{T}^{\prime \prime \prime}(\xi) \subset \mathcal{T}^{\prime \prime}(\xi) \subset \mathcal{T}^{\prime}(\xi) \subset \mathcal{T}(\xi)$ hold trivially, so that we have

$$
\tau^{\prime \prime \prime}(\xi) \leq \tau^{\prime \prime}(\xi) \leq \tau^{\prime}(\xi) \leq \tau(\xi)
$$

The main result of this section is the following chain of equalities, which summarizes Lemma 1, Theorem 1 and Theorem 2 .

Theorem 4. For any $\xi \in \mathbb{R} \backslash \mathbb{Q}$ we have

$$
\tau^{\prime \prime \prime}(\xi)=\tau^{\prime \prime}(\xi)=\tau^{\prime}(\xi)=\tau(\xi)=\frac{1}{\mu(\xi)-1} \in[0,1] .
$$

In particular the following assertions are equivalent: $\tau^{\prime \prime \prime}(\xi)=0, \tau^{\prime \prime}(\xi)=0, \tau^{\prime}(\xi)=$ $0, \tau(\xi)=0, \xi$ is a Liouville number.

As a corollary, we have $\tau^{\prime \prime \prime}(\xi)=\tau^{\prime \prime}(\xi)=\tau^{\prime}(\xi)=\tau(\xi)=1$ for almost all $\xi$ with respect to Lebesgue measure.

Proof. Since $2 \leq \mu(\xi) \leq+\infty$ for any $\xi \in \mathbb{R} \backslash \mathbb{Q}$, we have $\frac{1}{\mu(\xi)-1} \in[0,1]$.

For any $\tau \in \mathcal{T}(\xi)$, Proposition 1 yields $\mu(\xi) \leq 1+\frac{1}{\tau}$, that is, $\tau \leq \frac{1}{\mu(\xi)-1}$. If $\mathcal{T}(\xi) \neq \emptyset$, this gives $\mu(\xi)<\infty$ and $\tau(\xi) \leq \frac{1}{\mu(\xi)-1}$; this upper bound holds trivially if $\mathcal{T}(\xi)=\emptyset$.

By Eq. (4.1), we just have to prove that $\frac{1}{\mu(\xi)-1} \leq \tau^{\prime \prime \prime}(\xi)$ to finish the proof of Theorem 4. This is trivial if $\mu(\xi)=+\infty$. Otherwise, for any $\mu>\mu(\xi)$, Theorem 2 gives $\frac{1}{\mu-1} \in \mathcal{T}^{\prime \prime \prime}(\xi)$ so that $\frac{1}{\mu(\xi)-1} \leq \tau^{\prime \prime \prime}(\xi)$. This concludes the proof of Theorem 4

4.3. The multivariate case. Let $\xi_{0}, \ldots, \xi_{r}$ be real numbers, with $r \geq 1$. Throughout this section we assume

$$
\operatorname{dim}_{\mathbb{Q}} \operatorname{Span}_{\mathbb{Q}}\left(\xi_{0}, \ldots, \xi_{r}\right) \geq 2
$$

so that non-vanishing linear forms in $\xi_{0}, \ldots, \xi_{r}$ with integer coefficients can be arbitrarily small. We consider linear forms $L=\ell_{0} X_{0}+\cdots+\ell_{r} X_{r}$ with integer coefficients $\ell_{i}$, and we let $H(L)=\max _{0 \leq i \leq r}\left|\ell_{i}\right|$ and $L(\underline{\xi})=\ell_{0} \xi_{0}+\cdots+\ell_{r} \xi_{r}$, where $\underline{\xi}$ stands for the point $\left(\xi_{0}, \ldots, \xi_{r}\right)$ in $\mathbb{R}^{r+1}$.

Let us define the following sets:

- $\mathcal{T}_{r}(\underline{\xi})$ is the set of all $\tau>0$ for which there exists a sequence $\left(L_{n}\right)$ of linear forms with $L_{n}(\underline{\xi}) \neq 0$ for any $n$, and

$$
L_{n}(\underline{\xi}) \rightarrow 0, \quad\left|L_{n+1}(\underline{\xi})\right|=\left|L_{n}(\underline{\xi})\right|^{1+o(1)}, \quad \text { and } \quad\left|L_{n}(\underline{\xi})\right| \leq H\left(L_{n}\right)^{-\tau+o(1)} \text {. }
$$


- $\mathcal{T}_{r}^{\prime}(\underline{\xi})$ is the set of all $\tau>0$ for which there exists a sequence $\left(L_{n}\right)$ of linear forms, and $0<\alpha<1<\beta$, with

$$
\left|L_{n}(\underline{\xi})\right|^{1 / n} \rightarrow \alpha, \quad \limsup _{n \rightarrow+\infty} H\left(L_{n}\right)^{1 / n} \leq \beta, \quad \text { and } \quad \tau=\frac{-\log \alpha}{\log \beta} .
$$

- $\mathcal{T}_{r}^{\prime \prime}(\underline{\xi})$ is the set of all $\tau>0$ such that, for any increasing sequence $\left(Q_{n}\right)$ of positive integers, there exists a sequence $\left(L_{n}\right)$ of linear forms with

$$
H\left(L_{n}\right) \leq Q_{n}^{1+o(1)} \quad \text { and } \quad\left|L_{n}(\underline{\xi})\right|=Q_{n}^{-\tau+o(1)} .
$$

- $\mathcal{T}_{r}^{\prime \prime \prime}(\underline{\xi})$ is the set of all $\tau>0$ such that, for any sequences $\left(Q_{n}\right)$ and $\left(\varepsilon_{n}\right)$ of positive real numbers with

$$
\lim _{n \rightarrow+\infty} Q_{n}=+\infty, \quad \lim _{n \rightarrow+\infty} \varepsilon_{n}=0 \quad \text { and } \quad \varepsilon_{n} \geq Q_{n}^{-\tau+o(1)}
$$

there exists a sequence $\left(L_{n}\right)$ of linear forms with

$$
\lim _{n \rightarrow+\infty} \frac{H\left(L_{n}\right)}{Q_{n}}=\lim _{n \rightarrow+\infty} \frac{L_{n}(\underline{\xi})}{\varepsilon_{n}}=1 .
$$

Theorem 5 shows that $\tau \leq s$ for any $\tau$ in $\mathcal{T}_{r}(\underline{\xi})$, with $s=\operatorname{dim}_{\mathbb{Q}} \operatorname{Span}_{\mathbb{Q}}\left(\xi_{0}, \ldots, \xi_{r}\right)$ - 1 (and the same holds for $\mathcal{T}_{r}^{\prime}(\underline{\xi}), \mathcal{T}_{r}^{\prime \prime}(\underline{\xi})$ and $\mathcal{T}_{r}^{\prime \prime \prime}(\underline{\xi})$ ).

We let

$$
\tau_{r}(\underline{\xi})=\sup \mathcal{T}_{r}(\underline{\xi}),
$$

and in the same way, $\tau_{r}^{\prime}(\underline{\xi})=\sup \mathcal{T}_{r}^{\prime}(\underline{\xi}), \tau_{r}^{\prime \prime}(\underline{\xi})=\sup \mathcal{T}_{r}^{\prime \prime}(\underline{\xi}), \tau_{r}^{\prime \prime \prime}(\underline{\xi})=\sup \mathcal{T}_{r}^{\prime \prime \prime}(\underline{\xi})$, with the convention $\sup \emptyset=0$, so that each of $\tau_{r}(\underline{\xi}), \tau_{r}^{\prime}(\underline{\xi}), \tau_{r}^{\prime \prime}(\underline{\xi}), \bar{\tau}_{r}^{\prime \prime \prime}(\underline{\xi})$ belongs to $[0, s]$.

If we have $0<\tau<\tau^{\prime}$ and $\tau^{\prime} \in \mathcal{T}_{r}(\underline{\xi})$, then $\tau \in \mathcal{T}_{r}(\underline{\xi})$ so that $\mathcal{T}_{r}(\underline{\xi})$ is $\emptyset,\left(0, \tau_{r}(\underline{\xi})\right]$ or $\left(0, \tau_{r}(\underline{\xi})\right)$. The same holds for $\mathcal{T}_{r}^{\prime}(\underline{\xi}), \mathcal{T}_{r}^{\prime \prime}(\underline{\xi}), \mathcal{T}_{r}^{\prime \prime \prime}(\underline{\xi})$.

Moreover the inclusions $\mathcal{T}_{r}^{\prime \prime \prime}(\underline{\xi}) \subset \mathcal{T}_{r}^{\prime \prime}(\underline{\xi}) \subset \mathcal{T}_{r}^{\prime}(\underline{\xi}) \subset \mathcal{T}_{r}(\underline{\xi})$ hold trivially, so that we have

$$
\tau_{r}^{\prime \prime \prime}(\underline{\xi}) \leq \tau_{r}^{\prime \prime}(\underline{\xi}) \leq \tau_{r}^{\prime}(\underline{\xi}) \leq \tau_{r}(\underline{\xi}) .
$$

Let $\omega_{0}(\underline{\xi})$ be the supremum of the set of all $\omega>0$ such that there exist infinitely many $r+1$-tuples $\left(q_{0}, \ldots, q_{r}\right) \in \mathbb{Z}^{r+1}$ with

$$
\left|q_{i} \xi_{j}-q_{j} \xi_{i}\right| \leq \max \left(\left|q_{0}\right|, \ldots,\left|q_{r}\right|\right)^{-\omega} \text { for any } 1 \leq i<j \leq r .
$$

Up to renumbering $\xi_{0}, \ldots, \xi_{r}$, we may assume $\xi_{0} \neq 0$, and in this case we can replace (4.3) with

$$
\left|\frac{\xi_{j}}{\xi_{0}}-\frac{q_{j}}{q_{0}}\right| \leq\left|q_{0}\right|^{-\omega-1} \text { for any } i \in\{1, \ldots, r\}
$$

so that $\omega_{0}(\underline{\xi})$ measure the quality of simultaneous approximations to $\left(\xi_{1} / \xi_{0}, \ldots\right.$, $\left.\xi_{r} / \xi_{0}\right)$ by rational numbers with the same denominator.

When $r=1$ and $\xi_{0} \neq 0$, we have $\tau_{1}\left(\xi_{0}, \xi_{1}\right)=\tau\left(\xi_{1} / \xi_{0}\right)$ (and the analogous equalities for $\tau_{1}^{\prime}\left(\xi_{0}, \xi_{1}\right), \tau_{1}^{\prime \prime}\left(\xi_{0}, \xi_{1}\right)$ and $\left.\tau_{1}^{\prime \prime \prime}\left(\xi_{0}, \xi_{1}\right)\right)$, and $\omega_{0}\left(\xi_{0}, \xi_{1}\right)=\mu\left(\xi_{1} / \xi_{0}\right)-1$. This explains why the following result is a partial generalization of Theorem 4 .

Theorem 5. Let $\xi_{0}, \ldots, \xi_{r} \in \mathbb{R}$, with $r \geq 1$. Then we have

$$
\tau_{r}^{\prime \prime \prime}(\underline{\xi}) \leq \tau_{r}^{\prime \prime}(\underline{\xi}) \leq \tau_{r}^{\prime}(\underline{\xi}) \leq \tau_{r}(\underline{\xi}) \leq \frac{1}{\omega_{0}(\underline{\xi})} \leq s,
$$

under the assumption that $s=\operatorname{dim}_{\mathbb{Q}} \operatorname{Span}_{\mathbb{Q}}\left(\xi_{0}, \ldots, \xi_{r}\right)-1$ is positive. 
In the rest of this section, we prove this theorem and make some comments. The upper bounds $\tau_{r}^{\prime \prime \prime}(\underline{\xi}) \leq \tau_{r}^{\prime \prime}(\underline{\xi}) \leq \tau_{r}^{\prime}(\underline{\xi}) \leq \tau_{r}(\underline{\xi})$ hold trivially and have been observed before in (4.2).

Let us prove that $\tau_{r}(\underline{\xi}) \leq \frac{1}{\omega_{0}(\underline{\xi})}$. As above, we may assume that $\xi_{0}=1$. If $\tau_{r}(\underline{\xi})=0$, this result is trivial. Otherwise, let $0<\tau<\tau_{r}(\underline{\xi})$ and $0<\omega<\omega_{0}(\underline{\xi})$ (where $\omega_{0}(\underline{\xi})$ could be $+\infty$ ). Let $\left(L_{n}\right)$ be a sequence of linear forms such that $L_{n}(\underline{\xi}) \neq 0$ for any $n$, and

$$
L_{n}(\underline{\xi}) \rightarrow 0,\left|L_{n+1}(\underline{\xi})\right|=\left|L_{n}(\underline{\xi})\right|^{1+o(1)} \text {, and }\left|L_{n}(\underline{\xi})\right| \leq H\left(L_{n}\right)^{-\tau+o(1)} .
$$

There exist integers $q_{0}, \ldots, q_{r}$, with $\left|q_{0}\right|$ arbitrarily large, such that (4.4) holds. Let $n$ be the least positive integer such that $\left|q_{0} L_{n}(\underline{\xi})\right| \leq 1 / 2$. Taking $\left|q_{0}\right|$ sufficiently large ensures that $n$ can be made arbitrarily large. Since $n$ is defined in terms of $q_{0}$, any number denoted by $o(1)$ depends actually on $q_{0}$ and can be made arbitrarily small by choosing $\left|q_{0}\right|$ sufficiently large.

Since $n$ is the least positive integer such that $\left|q_{0} L_{n}(\underline{\xi})\right| \leq 1 / 2$, the integer $n+1$ does not satisfy this property, that is, $1 / 2<\left|q_{0}\right|\left|L_{n+1}(\underline{\xi})\right|=\left|q_{0}\right|\left|L_{n}(\underline{\xi})\right|^{1+o(1)}$, so that

$$
\left|q_{0}\right|=\left|L_{n}(\underline{\xi})\right|^{-1+o(1)} .
$$

Now we have (since $\xi_{0}=1$ )

$$
L_{n}\left(q_{0}, \ldots, q_{r}\right)=q_{0} L_{n}(\underline{\xi})+L_{n}\left(0, q_{1}-q_{0} \xi_{1}, \ldots, q_{r}-q_{0} \xi_{r}\right) .
$$

In the right-hand side, the first term has absolute value less than or equal to $1 / 2$, by choice of $n$. If the second term has absolute value less than the first one, then the integer $L_{n}\left(q_{0}, \ldots, q_{r}\right)$ has absolute value less than 1 so that it vanishes, and both terms in the right-hand side have the same absolute value, thereby contradicting the assumption.

Hence using also (4.6), (4.5) and (4.4), we have:

$$
\begin{aligned}
\left|L_{n}(\underline{\xi})\right|^{o(1)}=\left|q_{0} L_{n}(\underline{\xi})\right| & \leq\left|L_{n}\left(0, q_{1}-q_{0} \xi_{1}, \ldots, q_{r}-q_{0} \xi_{r}\right)\right| \\
& \leq r H\left(L_{n}\right) \max _{1 \leq i \leq r}\left|q_{i}-q_{0} \xi_{i}\right| \\
& \leq\left|L_{n}(\underline{\xi})\right|^{-\frac{1}{\tau}+o(1)}\left|q_{0}\right|^{-\omega}=\left|L_{n}(\underline{\xi})\right|^{\omega-\frac{1}{\tau}+o(1)} .
\end{aligned}
$$

Since $\lim _{n \rightarrow+\infty} \mid L_{n}(\underline{\xi)} \mid=0$ and $n$ can be chosen arbitrarily large, this implies $\omega \leq \frac{1}{\tau}$. This concludes the proof that $\tau_{r}(\underline{\xi}) \leq \frac{1}{\omega_{0}(\underline{\xi})}$.

Let us prove that $\frac{1}{\omega_{0}(\underline{\xi})} \leq s$, that is, $\omega_{0}(\underline{\xi}) \geq 1 / s$. Renumbering $\xi_{0}, \ldots, \xi_{r}$ if necessary, we may assume that $\xi_{0}, \ldots, \xi_{s}$ are linearly independent over the rationals; then $\xi_{s+1}, \ldots, \xi_{r}$ are linear combinations over $\mathbb{Q}$ of these numbers, and it is easy to check that $\omega_{0}\left(\xi_{0}, \ldots, \xi_{r}\right)=\omega_{0}\left(\xi_{0}, \ldots, \xi_{s}\right)$. Now the lower bound $\omega_{0}\left(\xi_{0}, \ldots, \xi_{s}\right) \geq$ $1 / s$ is a classical consequence of Dirichlet's pigeonhole principle. Indeed, for any positive integer $Q$, consider the $Q^{s}+1$ points $\left(\left\{q_{0} \xi_{1} / \xi_{0}\right\}, \ldots,\left\{q_{0} \xi_{s} / \xi_{0}\right\}\right) \in[0,1)^{s}$, for $0 \leq q_{0} \leq Q^{s}$ (here $\{x\}$ denotes the fractional part of a real number $x$ ), and the $Q^{s}$ cubes $\mathcal{C}_{i_{1}, \ldots, i_{s}}$ defined, for $0 \leq i_{1}, \ldots, i_{s}<Q$, by the inequalities $\frac{i_{1}}{Q} \leq x_{1}<\frac{i_{1}+1}{Q}$, $\ldots, \frac{i_{s}}{Q} \leq x_{s}<\frac{i_{s}+1}{Q}$. At least two of these points, given (say) by $q_{0}^{\prime}$ and $q_{0}^{\prime \prime}$, lie in the same cube. Letting $q_{0}=\left|q_{0}^{\prime}-q_{0}^{\prime \prime}\right|$ and denoting by $q_{j}$ the nearest integer to $q_{0} \xi_{j} / \xi_{0}$ for $j \in\{1, \ldots, s\}$, we obtain (4.4) with $\omega=1 / s$. Since a given $s+1$-tuple $\left(q_{0}, \ldots, q_{s}\right)$ is obtained in this way from only finitely many integers $Q$ (because $\xi_{0}, \ldots, \xi_{s}$ are 
Q-linearly independent), we obtain infinitely many tuples satisfying (4.4) so that $\omega_{0}\left(\xi_{0}, \ldots, \xi_{r}\right)=\omega_{0}\left(\xi_{0}, \ldots, \xi_{s}\right) \geq 1 / s$. This concludes the proof of Theorem 5 ,

A consequence of Theorem [5 is the inequality $\tau_{r}(\underline{\xi}) \leq s$, which amounts to the following statement, known as Nesterenko's linear independence criterion [10]:

Assume there exists a sequence $\left(L_{n}\right)$ of linear forms with $L_{n}(\underline{\xi}) \neq 0$ for any $n$ and

$L_{n}(\underline{\xi}) \rightarrow 0, \quad\left|L_{n+1}(\underline{\xi})\right|=\left|L_{n}(\underline{\xi})\right|^{1+o(1)}, \quad$ and $\quad\left|L_{n}(\underline{\xi})\right| \leq H\left(L_{n}\right)^{-\tau+o(1)}$

for some $\tau>0$. Then we have $\operatorname{dim}_{\mathbb{Q}} \operatorname{Span}_{\mathbb{Q}}\left(\xi_{0}, \ldots, \xi_{r}\right) \geq \tau+1$.

The above arguments provide a simple proof of this criterion (adapted from 7]), based on Dirichlet's pigeonhole principle and the upper bound $\tau_{r}(\underline{\xi}) \leq \frac{1}{\omega_{0}(\underline{\xi})}$ (which is also proved, essentially in the same way, as the first step in Nesterenko's inductive proof [10]).

In the one-dimensional case, where $\underline{\xi}=(1, \xi)$ with $\xi \in \mathbb{R} \backslash \mathbb{Q}$, the upper bound $\tau_{1}(\underline{\xi}) \leq \frac{1}{\omega_{0}(\underline{\xi})}$ corresponds to Proposition 1 , while $\frac{1}{\omega_{0}(\underline{\xi})} \leq 1$ simply means $\mu(\xi) \geq 2$ (and one way to prove this fact is to use Dirichlet's pigeonhole principle as in the multivariate setting).

It would be very interesting to investigate further around Theorem 5 , for instance to know for which $\underline{\xi}$ equality holds (as in the univariate case of Theorem 4). Is it the case for almost all $\underline{\xi}$ with respect to Lebesgue measure? It is well known that $\frac{1}{\omega_{0}(\underline{\xi})}=s=r$ for almost all $\underline{\xi}$.

Another question worth studying is the connection between $\tau_{r}(\underline{\xi}), \tau_{r}^{\prime}(\underline{\xi}), \tau_{r}^{\prime \prime}(\underline{\xi})$, $\tau_{r}^{\prime \prime \prime}(\underline{\xi})$, and the exponent $\omega_{k}(\underline{\xi})$ that measures the distance of $\underline{\xi}=\left(\xi_{0}, \ldots, \xi_{r}\right)$ to subspaces of dimension $k+1$ of $\mathbb{R}^{r+1}$ defined over $\mathbb{Q}$, for $k<s$ (see [12, [9], 4] and [10]).

At last, many other questions may be asked about these exponents, for instance how to understand the set of values taken by $\tau_{r}(\underline{\xi})\left(\operatorname{resp} . \tau_{r}^{\prime}(\underline{\xi}), \tau_{r}^{\prime \prime}(\underline{\xi}), \tau_{r}^{\prime \prime \prime}(\underline{\xi})\right.$ ) as $\underline{\xi}$ varies, especially when $\underline{\xi}$ is assumed to be of a special form (for instance $\underline{\xi}=\left(1, \xi, \ldots, \xi^{r}\right)$ with $\left.\xi \in \mathbb{R} \backslash \mathbb{Q}\right)$. It would be interesting to study if there is any connection with Mahler's and Koksma's classifications of numbers.

\section{REFERENCES}

[1] B. Adamczewski, "Sur l'exposant de densité des nombres algébriques", International Math. Research Notices (2007), Article ID 024, 6 pages. MR.2345344 (2008f:11080)

[2] R. Apéry, "Irrationalité de $\zeta(2)$ et $\zeta(3)$ ", in: Journées Arithmétiques (Luminy, 1978), Astérisque 61 (1979), pp. 11-13.

[3] K. Ball and T. Rivoal, "Irrationalité d'une infinité de valeurs de la fonction zêta aux entiers impairs", Invent. Math. 146 (2001), no. 1, pp. 193-207. MR.1859021 (2003a:11086)

[4] Y. Bugeaud and M. Laurent, "On transfer inequalities in Diophantine approximation, II", Math. Zeitschrift, to appear.

[5] S. Fischler, "Restricted rational approximation and Apéry-type constructions", Indag. Math., to appear.

[6] S. Fischler and T. Rivoal, "Un exposant de densité en approximation rationnelle", International Math. Research Notices (2006), no. 24, Article ID 95418, 48 pages. MR2272100 (2007h:11079)

[7] S. Fischler and W. Zudilin, "A refinement of Nesterenko's linear independence criterion with applications to zeta values", MPIM preprint 2009-35, May 2009, Math. Ann., to appear, available from www.mpim-bonn.mpg.de/preprints/send?bid $=4020$.

[8] M. Kontsevich and D. Zagier, "Periods", in: Mathematics unlimited-2001 and beyond, Springer, 2001, pp. 771-808. MR.1852188(2002i:11002) 
[9] M. Laurent, "On transfer inequalities in Diophantine approximation", in: Analytic number theory, Essays in honour of Klaus Roth, Cambridge Univ. Press, 2009, pp. 306-314. MR2508652

[10] Y. Nesterenko, "On the linear independence of numbers", Vestnik Moskov. Univ. Ser. I Mat. Mekh. [Moscow Univ. Math. Bull.] 40 (1985), no. 1, pp. 46-49 [69-74]. MR783238(86j:11074)

[11] T. Rivoal, "La fonction zêta de Riemann prend une infinité de valeurs irrationnelles aux entiers impairs", C. R. Acad. Sci. Paris, Ser. I 331 (2000), no. 4, pp. 267-270. MR1787183 (2001k:11138)

[12] W. Schmidt, "On heights of algebraic subspaces and Diophantine approximations", Annals of Math. (2) 85 (1967), pp. 430-472. MR0213301 (35:4165)

Université Paris-Sud, Laboratoire de Mathématiques d’Orsay, Orsay Cedex, F-91405, France - AND - CNRS, Orsay CEDEx, F-91405, France

Institut Fourier, CNRS UMR 5582, Université Grenoble 1, 100 Rue Des Maths, BP 74, 38402 Saint-Martin d'Hères Cedex, France 